\title{
Level Crossing Analysis of Growing surfaces
}

\author{
F. Shahbazi, ${ }^{a}$, S. Sobhanian, ${ }^{b, e}$, M. Reza Rahimi Tabar ${ }^{c, d, e}$, S. Khorram ${ }^{f}$, \\ G.R. Frootan, ${ }^{b}$ and H. Zahed, ${ }^{b}$ \\ ${ }^{a}$ Dept. of Physics, Sharif University of Technology, P.O.Box 11365-9161, Tehran, Iran. \\ ${ }^{b}$ Dep. of Theoretical Physics and Astrophysics, Tabriz University, Tabriz 51664, Iran \\ ${ }^{c}$ CNRS UMR 6529, Observatoire de la Côte d'Azur, BP 4229, 06304 Nice Cedex 4, France, \\ ${ }^{d}$ Dept. of Physics, IUST, P.O.Box 16844, Tehran, Iran. \\ e Research Institute for Fundamental Sciences, Tabriz 51664, Iran. \\ ${ }^{f}$ Center for Applied and Astronomical Research, Physics Departmant, \\ Tabriz Univrsity-51664, Tabriz Iran.
}

\begin{abstract}
We investigate the average frequency of positive slope $\nu_{\alpha}^{+}$, crossing the height $\alpha=h-\bar{h}$ in the surface growing processes. The exact level crossing analysis of the random deposition model and the Kardar-Parisi-Zhang equation in the strong coupling limit before creation of singularities are given. PACS: 52.75.Rx, 68.35.Ct.
\end{abstract}

\section{INTRODUCTION}

Due to the technical importance and fundamental interest, a great deal of effort has been devoted to understanding the mechanism of thin-film growth and the kinetic roughening of growing surfaces in various growth techniques. Analytical and numerical treatments of simple growth models suggest, quite generally, the height fluctuations have a self-similar character and their average correlations exhibit a dynamic scaling form [1-6]. It is known that to derive the quantitative information of the surface morphology one may consider a sample of size $L$ and define the mean height of growing film $\bar{h}$ and its roughness $w$ by [1]:

$$
\bar{h}(L, t)=\frac{1}{L} \int_{-L / 2}^{L / 2} d x h(x, t)
$$

and

$$
w(L, t)=\left(\left\langle(h-\bar{h})^{2}\right\rangle\right)^{1 / 2},
$$

where $\langle\cdots\rangle$ denotes an averaging over different realization (samples). Starting from a flat interface (one of the possible initial conditions), it was conjectured by Family and Vicsek [7] that a scaling of space by factor $b$ and of time by a factor $b^{z}$ ( $z$ is the dynamical scaling exponent), re-scales the roughness $w$ by factor $b^{\chi}$ as follows: $w\left(b L, b^{z} t\right)=b^{\chi} w(L, t)$, which implies that

$$
w(L, t)=L^{\chi} f\left(t / L^{z}\right) .
$$

If for large $t$ and fixed $L\left(t / L^{z} \rightarrow \infty\right), w$ saturate then $f(x) \rightarrow$ const., as $x \rightarrow \infty$. However, for fixed large $L$ and $1<<t<<L^{z}$, one expects that correlations of the height fluctuations are set up only within a distance $t^{1 / z}$ and thus must be independent of $L$. This implies that for $x<<1, f(x) \sim x^{\beta}$ with $\beta=\chi / z$. Thus dynamic scaling postulates that, $w(L, t) \sim t^{\beta}$ for $1<<t<<L^{z}$ and $\sim L^{\chi}$ for $t>>L^{z}$. The roughness exponent $\chi$ and the dynamic exponent $z$ characterize the self-affine geometry of the surface and its dynamics, respectively.

Here we introduce the level crossing analysis in the context of surface growth processes. In the level crossing analysis we are interested in determining the average frequency ( in spatial dimension ) of observing of the definite value for height function $h-\bar{h}=\alpha$ in growing thin films, $\nu_{\alpha}^{+}$, from which one can find the averaged number of crossing the given height in sample with size L. The average number of visiting the height $h-\bar{h}=\alpha$ with positive slop will be $N_{\alpha}^{+}=\nu_{\alpha}^{+} L$. It can be shown that the $\nu_{\alpha}^{+}$can be written in terms of joint probability distribution function (PDF) of $h-\bar{h}$ and its gradient. Therefore the quantity $\nu_{\alpha}^{+}$carry the whole information of surface which lies in joint PDF of height and its gradient fluctuations. This work aims to study the frequency of positive slope crossing (i.e. $\nu_{\alpha}^{+}$) in time $t$ on the growing surface in a sample with size L. We introduce a quantity $N_{\text {tot }}^{+}$ which is defined as $N_{\text {tot }}^{+}=\int_{-\infty}^{+\infty} \nu_{\alpha}^{+} d \alpha$ to measure the total number of crossing the surface with positive slop. The $N_{\text {tot }}^{+}$and the path which is constructed by growing surface are in the same order. It is expected that in the stationary state the $N_{\text {tot }}^{+}$to become size dependent. We determine the time and height dependence of $\nu_{\alpha}^{+}$for two exactly solvable models, random deposition model (RD) and Kardar-Parisi-Zhang (KPZ) equation in the strong coupling limit and before creation of singularities (sharp valleys) with short range forcing. It is shown that the RD-model and KPZ equation have different $\nu_{\alpha}^{+}$but $N_{t o t}^{+}$ scales with time as $t^{1 / 2}$ in both models.

In section 2 we discuss the connection between $\nu_{\alpha}^{+}$and underlying probability distribution functions (PDF) of growing surfaces. Exact expression of $\nu_{\alpha}^{+}$for the RD model and with short-range forcing is given in section 3 . In section 4 we derive the integral representation of $\nu_{\alpha}^{+}$for the KPZ equation in $1+1$ dimension and in the strong coupling limit before the creation of singularities. We summarize the results in section 5 . 


\section{THE LEVEL CROSSING ANALYSIS OF GROWING SURFACE}

Consider a sample function of an ensemble of functions which make up the homogeneous random process $h(x, t)$. Let $n_{\alpha}^{+}$denote the number of positive slope crossing of $h(x)-\bar{h}=\alpha$ in time $t$ for a typical sample size $L$ (see figure 1$)$ and let the mean value for all the samples be $N_{\alpha}^{+}(L)$ where

$$
N_{\alpha}^{+}(L)=E\left[n_{\alpha}^{+}(L)\right] .
$$

Since the process is homogeneous, if we take a second interval of $L$ immediately following the first we shall obtain the same result, and for the two intervals together we shall therefore obtain

$$
N_{\alpha}^{+}(2 L)=2 N_{\alpha}^{+}(L),
$$

from which it follows that, for a homogeneous process, the average number of crossing is proportional to the space interval $L$. Hence

$$
N_{\alpha}^{+}(L) \propto L,
$$

or

$$
N_{\alpha}^{+}(L)=\nu_{\alpha}^{+} L \text {. }
$$

which $\nu_{\alpha}^{+}$is the average frequency of positive slope crossing of the level $h-\bar{h}=\alpha$. We now consider how the frequency parameter $\nu_{\alpha}^{+}$can be deduced from the underlying probability distributions for $h-\bar{h}$. Consider a small length $d l$ of a typical sample function. Since we are assuming that the process $h-\bar{h}$ is a smooth function of $x$, with no sudden ups and downs, if $d l$ is small enough, the sample can only cross $h-\bar{h}=\alpha$ with positive slope if $h-\bar{h}<\alpha$ at the beginning of the interval location $x$. Furthermore there is a minimum slope at position $x$ if the level $h-\bar{h}=\alpha$ is to be crossed in interval $d l$ depending on the value of $h-\bar{h}$ at location $x$. So there will be a positive crossing of $h-\bar{h}=\alpha$ in the next space interval $d l$ if, at position $x$,

$$
h-\bar{h}<\alpha \quad \text { and } \quad \frac{d(h-\bar{h})}{d l}>\frac{\alpha-(h-\bar{h})}{d l} .
$$

Actually what we really mean is that there will be high probability of a crossing in interval $d l$ if these conditions are satisfied $[8,9]$.

In order to determine whether the above conditions are satisfied at any arbitrary location $x$, we must find how the values of $y=h-\bar{h}$ and $y^{\prime}=\frac{d y}{d l}$ are distributed by considering their joint probability density $p\left(y, y^{\prime}\right)$. Suppose that the level $y=\alpha$ and interval $d l$ are specified. Then we are only interested in values of $y<\alpha$ and values of $y^{\prime}=\left(\frac{d y}{d l}\right)>\frac{\alpha-y}{d l}$, which means that the region between the lines $y=\alpha$ and $y^{\prime}=\frac{\alpha-y}{d l}$ in the plane $\left(y, y^{\prime}\right)$.

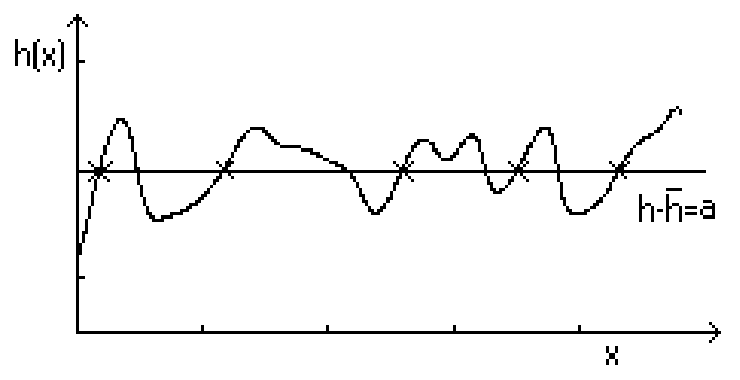

FIG. 1. positive slope crossing of the level $h-\bar{h}=\alpha$.

Hence the probability of positive slope crossing of $y=\alpha$ in $d l$ is:

$$
\int_{0}^{\infty} d y^{\prime} \int_{\alpha-y^{\prime} d l}^{\alpha} d y p\left(y, y^{\prime}\right)
$$

When $d l \rightarrow 0$, it is legitimate to put

$$
p\left(y, y^{\prime}\right)=p\left(y=\alpha, y^{\prime}\right)
$$

Since at large values of $y$ and $y^{\prime}$ the probability density function approaches zero fast enough, therefore eq.(6) may be written as:

$$
\int_{0}^{\infty} d y^{\prime} \int_{\alpha-y^{\prime} d l}^{\alpha} d y p\left(y=\alpha, y^{\prime}\right)
$$

in which the integrand is no longer a function of $y$ so that the first integral is just: $\int_{\alpha-y^{\prime} d l}^{\alpha} d y p\left(y=\alpha, y^{\prime}\right)=$ $p\left(y=\alpha, y^{\prime}\right) y^{\prime} d l$, so that the probability of slope crossing of $y=\alpha$ in $d l$ is equal to:

$$
d l \int_{0}^{\infty} p\left(\alpha, y^{\prime}\right) y^{\prime} d y^{\prime}
$$

in which the term $p\left(\alpha, y^{\prime}\right)$ is the joint probability density $p\left(y, y^{\prime}\right)$ evaluated at $y=\alpha$.

We have said that the average number of positive slope crossing in scale $L$ is $\nu_{\alpha}^{+} L$, according to (7). The average number of crossing in interval $d l$ if therefore $\nu_{\alpha}^{+} d l$. So average number of positive crossings of $y=\alpha$ in interval $d l$ is equal to the probability of positive crossing of $y=$ $a$ in $d l$, which is only true because $d l$ is small and the process $y(x)$ is smooth so that there cannot be more than one crossing of $y=\alpha$ in space interval $d l$, Therefore we have $\nu_{\alpha}^{+} d l=d l \int_{0}^{\infty} p\left(\alpha, y^{\prime}\right) y^{\prime} d y^{\prime}$, from which we get the following result for the frequency parameter $\nu_{\alpha}^{+}$in terms of the joint probability density function $p\left(y, y^{\prime}\right)$

$$
\nu_{\alpha}^{+}=\int_{0}^{\infty} p\left(\alpha, y^{\prime}\right) y^{\prime} d y^{\prime} .
$$

In the following sections we are going to derive the $\nu_{\alpha}^{+}$ via the joint PDF of $h-\bar{h}$ and height gradient. To derive the joint PDF we use the master equation method [10-11]. This method enables us find the $\nu_{\alpha}^{+}$in terms of generating function $Z(\lambda, \mu, x, t)=\langle\exp (-i \lambda(h(x, t)-\bar{h})-i \mu u(x, t))\rangle$, where $u(x, t)=-\nabla h$. 


\section{THE FREQUENCY OF A DEFINITE HEIGHT WITH POSITIVE SLOPE FOR THE RANDOM DEPOSITION MODEL}

In random deposition model particles are dropped randomly over deposition sites, and stick to the top of the pre-existing column on the site [ 1 ]. The height of each column thus performs an independent random walk. This model leads to unrealistically rough surface whose overall width growth with the exponent $\beta=\frac{1}{2}$ without saturation. In the continuum limit the random deposition model is described by the following equations;

$$
\frac{\partial}{\partial t} h(x, t)=f(x, t) \quad \frac{\partial}{\partial t} u(x, t)=f_{x}
$$

where $h(x, t)$ is the height field, $u(x, t)=\frac{\partial}{\partial x} h(x, t)$ and $f(x, t)$ is a zero mean random force gaussian correlated in space and white in time,

$$
\left\langle f(x, t) f\left(x^{\prime}, t^{\prime}\right)\right\rangle=2 D_{0} D\left(x-x^{\prime}\right) \delta\left(t-t^{\prime}\right)
$$

where $D(x)$ is space correlation function and is an even function of its argument. It has the following form;

$$
D\left(x-x^{\prime}\right)=\frac{1}{\sqrt{\pi} \sigma} \exp \left(-\frac{\left(x-x^{\prime}\right)^{2}}{\sigma^{2}}\right)
$$

where $\sigma$ is the standard deviation of $D\left(x-x^{\prime}\right)$. The average force on the interface is not essential and can be removed by a simple shift of $h$ ( i.e $h \rightarrow(h-\bar{F} t)$ where $\bar{F}=<f(x, t)>)$. Typically the correlation of forcing is considered as delta function for mimicking the short range correlation. We regularize the delta function correlation by a gaussian function. When the variance $\sigma$ is much less than the system size we would expect that the model would represent a short range character for the forcing. So we would stress that our calculations are done for finite $\sigma \ll L$, where $L$ is the system size. The parameters $D_{0}$ is describing the noise strength.

Now assuming the homogeneity we define the generating function as:

$$
Z(\lambda, \mu, t)=\langle\exp (-i \lambda h(x, t)-i \mu u(x, t)\rangle
$$

by using the eq.(14) we can find the following equation for the evolution of $Z(\lambda, \mu, t)$ :

$$
\begin{aligned}
\frac{\partial}{\partial t} Z(\lambda, \mu, t)= & -i \lambda\left\langle h_{t}(x, t) \exp (-i \lambda h(x, t)-i \mu u(x, t)\rangle\right. \\
& -i \mu\left\langle u_{t}(x, t) \exp (-i \lambda h(x, t)-i \mu u(x, t)\rangle\right. \\
= & -i \lambda\left\langle f(x, t) \exp \left(-i \lambda h(x, t)-i \mu f_{x}(x, t)\right\rangle\right. \\
& -i \mu\left\langle u_{t} \exp (-i \lambda h(x, t)-i \mu u(x, t)\rangle\right. \\
= & -\lambda^{2} D_{0} D(0) Z+\mu^{2} D_{0} D_{x x}(0) Z
\end{aligned}
$$

The joint probability density function of $h$ and $u$ can be obtain by fourier transform of the generating function:

$$
P(h, u, t)=\frac{1}{2 \pi} \int d \lambda d \mu e^{i \lambda h+i \mu u} Z(\lambda, \mu, t),
$$

so by fourier transforming of the eq.(18) we get the Fokker-Planck equation as:

$$
\frac{\partial}{\partial t} P=D_{0} D(0) \frac{\partial^{2}}{\partial h^{2}} P-D_{0} D_{x x}(0) \frac{\partial^{2}}{\partial u^{2}} P .
$$

the solution of the above equation can be separated as $P(h, u, t)=p_{1}(h, t) p_{2}(u, t)$. Using the initial conditions $P_{1}(h, 0)=\delta(h)$ and $P_{2}(u, 0)=\delta(u)$ (starting from flat surface) it can be shown that:

$$
\begin{aligned}
P(h, u, t)= & \frac{1}{4 \pi t \sqrt{-D_{0}^{2} D(0) D_{x x}(0)}} \\
& \exp \left(-\frac{h^{2}}{4 D_{0} D(0) t}+\frac{u^{2}}{4 D_{0} D_{x x}(0) t}\right),
\end{aligned}
$$

from which the frequency of repeating a definite height $(h(x, t)=\alpha)$ can be calculated as

$$
\begin{aligned}
\nu_{\alpha}^{+} & =\int_{0}^{\infty} u P(\alpha, u) d u \\
& =\frac{1}{2 \pi} \sqrt{-\frac{D_{x x}(0)}{D(0)}} \exp \left(-\frac{\alpha^{2}}{4 D(0) t}\right) \\
& =\frac{1}{2 \pi \sigma} \exp \left(-\frac{\alpha^{2}}{4 D_{0} D(0) t}\right)
\end{aligned}
$$

The quantity $\nu_{\alpha}^{+}$in RD model has a gaussian form with respect to $\alpha$. The zero level crossing scales with $\sigma$ as $\nu_{\alpha=0}^{+} \sim \sigma^{-1}$. Also using the eq.(22) it is found that $N_{\text {tot }}^{+}=D_{0}^{1 / 2} \pi^{-3 / 4} \sigma^{-3 / 2} t^{1 / 2}$. This shows that there is no stationary state for the RD model and the quantity $N_{t o t}^{+}$ diverges without saturation.

\section{FREQUENCY OF A DEFINITE HEIGHT WITH POSITIVE SLOPE FOR KPZ EQUATION BEFORE THE SINGULARITY FORMATION}

In the Kardar-Parisi-Zhang (KPZ) model (e.g. in the 1-dimension), the surface height $h(x, t)$ on the top of location $x$ of 1-dimensional substrate satisfies a stochastic random equation:

$$
\frac{\partial h}{\partial t}-\frac{\alpha}{2}\left(\partial_{x} h\right)^{2}=\nu \partial_{x}^{2} h+f(x, t) .
$$

where $\alpha \geq 0$ and $f$ is a zero-mean, statistically homogeneous, white in time and gaussian process with covariance as eq.(16). The parameters $\nu, \alpha$ and $D_{0}$ ( and $\sigma$ ) are describing surface relaxation, lateral growth and the noise strength, respectively. Let us define the generating 


$$
Z(\lambda, \mu, x, t)=\langle\exp (-i \lambda(h(x, t)-\bar{h})-i \mu u(x, t))\rangle .
$$

Where $u(x, t)=-\partial_{x} h(x, t)$. Assuming statistical homogeneity i.e. $Z_{x}=0$ it follows from eq.(23) that $Z$ satisfies the following equation;

$$
\begin{aligned}
& -i \mu Z_{t}=\gamma(t) \lambda \mu Z-\frac{\alpha}{2} \lambda \mu Z_{\mu \mu}+i \lambda^{2} \mu k(0) Z \\
& -i \mu^{3} k_{x x}(0) Z-i\left(\nu \lambda^{2}+i \alpha \lambda\right) Z_{\mu} \\
& -\mu^{2} \nu\left\langle u_{x x}(x, t) \exp (-i \lambda \tilde{h}(x, t)-i \mu u(x, t))\right\rangle .
\end{aligned}
$$

where $k\left(x-x^{\prime}\right)=2 D_{0} D\left(x-x^{\prime}\right), \gamma(t)=\bar{h}_{t}, k(0)=\frac{D_{0}}{\sqrt{\pi} \sigma}$ and $k_{x x}(0)=-\frac{2 D_{0}}{\sqrt{\pi} \sigma^{3}}$. and $\tilde{h}(x, t)=h(x, t)-\bar{h}$. Once trying to develop the statistical theory of the roughened surface it becomes clear that the inter-dependency of the height difference and height gradient statistics would be taken into account. The very existence of the non-linear term in the KPZ equation leads to development of the cusp singularities in a finite time and in the strong coupling limit i.e. $\nu \rightarrow 0$. So one would distinguish between different time regimes. Recently it has been shown that starting from the flat interface the KPZ equation will develop sharp valleys singularity after time scale $t *$, where $t *$ depends on the forcing properties as $t *=\left(\frac{1}{4}\right)^{4 / 3}(\pi)^{1 / 6} D_{0}{ }^{-1 / 3} \alpha^{-2 / 3} \sigma^{5 / 3}[10]$. This means that for time scales before $t *$ the relaxation contribution tends to zero when $\nu \rightarrow 0$. In this regime one can observe that the generating function equation is closed. The solutions of the resulting equation is easily derived, ( starting from a flat surface, i.e. $h(x, 0)=0$ and $u(x, 0)=0)$, and has the following form [10];

$$
\begin{aligned}
Z(\mu, \lambda, t)=\left(1-\tanh ^{2}\left(\sqrt{2 i k_{x x}(0) \alpha \lambda} t\right)\right) \\
\exp \left[-\frac{5}{8} \ln \left(1-\tanh ^{4}\left(\sqrt{2 i k_{x x}(0) \alpha \lambda} t\right)\right)\right. \\
+\frac{5}{4} \tanh ^{-1}\left(\tanh ^{2}\left(\sqrt{2 i k_{x x}(0) \alpha \lambda} t\right)\right)-\lambda^{2} k(0) t \\
-\frac{1}{16} \ln ^{2}\left(\frac{1-\tanh \left(\sqrt{2 i k_{x x}(0) \alpha \lambda} t\right)}{1+\tanh \left(\sqrt{2 i k_{x x}(0) \alpha \lambda}\right)}\right) \\
\left.-\frac{1}{2} i \mu^{2} \sqrt{\frac{2 i k_{x x}(0)}{\alpha \lambda}} \tanh \left(\sqrt{2 i k_{x x}(0) \alpha \lambda} t\right)\right] .
\end{aligned}
$$

One can construct $P(\tilde{h}, u, t)$ in terms of generating function $Z$ as eq.(19), from which the frequency of repeating a definite height $(h(x, t)-\bar{h}=\alpha)$ with positive slope can be calculated as $\nu_{\alpha}^{+}=\int_{0}^{\infty} u P(\alpha, u) d u$.

In fig. 2 we plot the $\nu_{\alpha}^{+}$for time scales before creation of singularity, $t / t *=0.05,0.15$ and 0.25 . In the KPZ equation due to the nonlinear term there is no $h \rightarrow-h$ symmetry and one can deduce that the $\nu_{\alpha}^{+}$is also not symmetric under $h \rightarrow-h$.

To derive the $N_{\text {tot }}^{+}$let us express the $\nu_{\alpha}^{+}$and $N_{\text {tot }}^{+}$in terms of the generating function Z. It can be easily shown

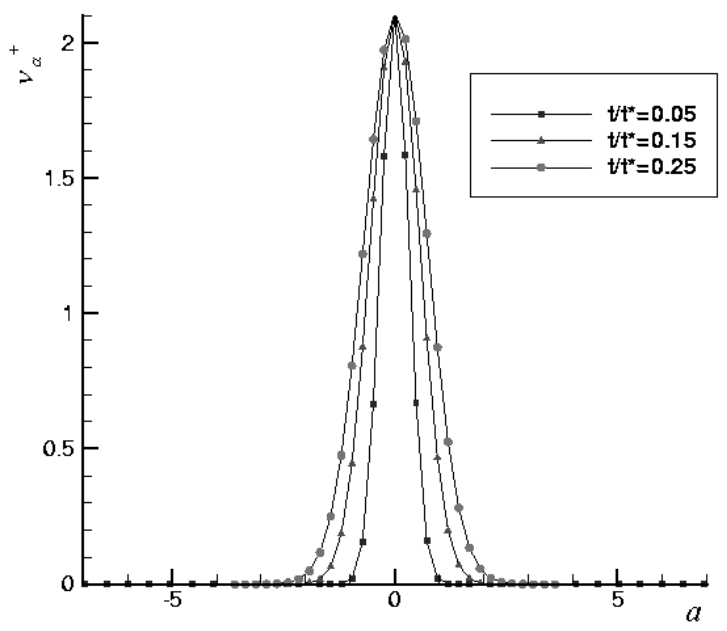

FIG. 2. plot of $\nu_{\alpha}^{+}$vs $\alpha$ for the KPZ equation in the strong coupling and befor the creation of sharp vallyes for time scale $t / t *=0.05,0.15$ and 0.25 .

that the $\nu_{\alpha}^{+}$and $N_{t o t}^{+}$can be written in terms of the generating function $Z$ as:

$$
\nu_{\alpha}^{+}=\frac{1}{2 \pi} \int_{-\infty}^{+\infty} \int_{-\infty}^{+\infty}-\frac{1}{\mu^{2}} Z(\lambda, \mu) \exp (i \lambda \alpha) d \lambda d \mu
$$

and

$$
N_{t o t}^{+}=\int_{-\infty}^{+\infty}-\frac{1}{\mu^{2}} Z(\lambda \rightarrow 0, \mu) d \lambda
$$

Using the eq.( 25 ) one finds $N_{\text {tot }}^{+} \sim \sigma^{-3 / 2} t^{1 / 2}$. We note that the expression of the $\nu_{\alpha}^{+}$for the RD model and KPZ equation before $t *$ are different functions of $\alpha$ but $N_{\text {tot }}^{+}$scales as $\sim \sigma^{-3 / 2} t^{1 / 2}$ in both models. In fig.(3) using the direct numerical integration of joint PDF of height and its gradient we plot the $N_{t o t}$ vs $t$. In this gragh $N_{t o t} \sim t^{1 / 2}$ which is in agreement with analytical prediction. 


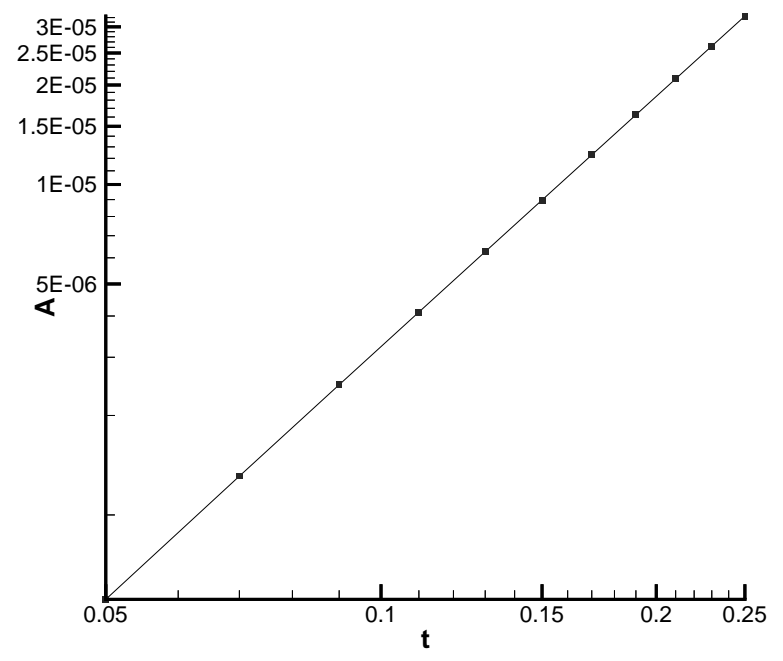

FIG. 3. log-log plot of $N_{\text {tot }}$ (A) vs $t$ for the KPZ equation in the strong coupling before the creation of sharp vallyes.

\section{CONCLUSION}

We obtained some results in the problems of RD model and KPZ equation in $1+1$ dimensions with a Gaussian forcing which is white in time and short range correlated in space. We determined the explicit expression of average frequency of crossing i.e. $\nu_{\alpha}^{+}$of observing of the definite value for height function $h-\bar{h}=\alpha$ in a growing thin films for the RD model, from which one can find the averaged number of crossing the given height in a sample with size L. It is shown the $\nu_{\alpha}^{+}$is symmetric under $h \rightarrow-h$. The integral representation of $\nu_{\alpha}^{+}$is given for the KPZ equation in the strong coupling limit before the creation of sharp valleys. We introduced the quantity $N_{\text {tot }}^{+}=\int_{-\infty}^{+\infty} \nu_{\alpha}^{+} d \alpha$, which measures the total number of positive crossing of growing surface and show that for the $\mathrm{RD}$-model and the KPZ equation in the strong coupling limit and before the creation of sharp valleys $N_{\text {tot }}^{+}$scales as $\sigma^{-3 / 2} t^{1 / 2}$. It is noted that for these models $\nu_{\alpha}^{+}$has different expression in terms of $\alpha$. The ideas presented in this paper can be used to find the $\nu_{\alpha}^{+}$of the general Langevin equation with given drift and diffusion coefficients.

Acknowledgment We would like to thank F. Aazami for useful comments. This work supported by Research Institute for Fundamental Sciences. in Surface Growth" (Cambridge University Press, New York, 1995).

[2] T. Halpin-Healy and Y. C. Zhang, Phys. Rep.245,218(1995);J. Krug, Adv. Phys.46,139(1997)

[3] J. Krug and H. Spohn in "Solids Far from Equilibrium Growth, Morphology and Defects", edited by C. Godreche (Cambridge University Press, New York, 1990).

[4] P. Meakin, "Fractal, Scaling and Growth Far from Equilibrium" (Cambridge University Press, Cambridge, 1998).

[5] M. Marsilli, A. Maritan, F. Toigoend J.R. Banavar,Rev. Mod. Phys., 68,963 (1996)

[6] M. Kardar, Physica A 281,295(2000).

[7] F. Family and T. Vicsek,J.Phys.A 18,L75(1985)

[8] S.O. Rice " Mathematical Analysis of Random Noise" Bell System Tech. J. Vol.23,(1944),282; Vol. 24, (1945), 46

[9] D.E. Newland " An Introduction to Random Vibration, Spectral and Wavelet Analysis" ( Longman, Harlow and Wiley, New York, 1993 )

[10] A. A. Masoudi, F. Shahbazi, J. Davoudi and M. Reza Rahimi Tabar, Phys. Rev. E 65, 026132(2002). 